\title{
Les pratiques pédagogiques " exemplaires » en sciences de l'information
}

\section{The Best Pedagogical Practices in Information Sciences \\ Las "mejores" prácticas pedagógicas en ciencias de la información}

\section{Zeïneb Gharbi et Dominique Maurel}

Volume 53, numéro 1, janvier-mars 2007

URI : https://id.erudit.org/iderudit/1029214ar

DOI : https://doi.org/10.7202/1029214ar

Aller au sommaire du numéro

Éditeur(s)

Association pour l'avancement des sciences et des techniques de la documentation (ASTED)

ISSN

0315-2340 (imprimé)

2291-8949 (numérique)

Découvrir la revue

Citer cet article

Gharbi, Z. \& Maurel, D. (2007). Les pratiques pédagogiques « exemplaires » en sciences de l'information. Documentation et bibliothèques, 53(1), 15-26. https://doi.org/10.7202/1029214ar
Résumé de l'article

L'article s’intéresse aux pratiques pédagogiques « exemplaires » en sciences de l'information. Après une mise en contexte et quelques précisions d'ordre méthodologique, nous présentons une recension des écrits consacrés à ce sujet en trois volets : des réflexions théoriques sur les pratiques pédagogiques en sciences de l'information, des guides proposant des approches d'enseignement, et des bilans d'expérience rapportés par des enseignants. Nous dressons ensuite l'inventaire des pratiques pédagogiques « exemplaires » identifiées par les enseignants de l'École de bibliothéconomie et des sciences de l'information (EBSI). Enfin, nous tirons quelques constats à partir de la littérature recensée et de l'inventaire effectué.
Tous droits réservés (c) Association pour l'avancement des sciences et des techniques de la documentation (ASTED), 2007
Ce document est protégé par la loi sur le droit d'auteur. L'utilisation des services d’Érudit (y compris la reproduction) est assujettie à sa politique d'utilisation que vous pouvez consulter en ligne.

https://apropos.erudit.org/fr/usagers/politique-dutilisation/ 


\title{
Les pratiques pédagogiques «exemplaires » en sciences de l'information
}

\author{
ZEÏNEB GHARBI, PH.D. \\ Stagiaire postdoctorale \\ Centre de recherche Interdisciplinaire sur les technologies émergentes (CITÉ) \\ Université de Montréal \\ zeineb.gharbi@umontreal.ca \\ DOMINIQUE MAUREL, PH.D. \\ Stagiaire postdoctorale \\ Faculty of Information Studies \\ University of Toronto \\ dominique.maurel@utoronto.ca
}

\section{RÉSUmé | ABSTRACTS | RESUMEN}

L'article s'intéresse aux pratiques pédagogiques «exemplaires» en sciences de l'information. Après une mise en contexte et quelques précisions d'ordre méthodologique, nous présentons une recension des écrits consacrés à ce sujet en trois volets: des réflexions théoriques sur les pratiques pédagogiques en sciences de l'information, des guides proposant des approches d'enseignement, et des bilans d'expérience rapportés par des enseignants. Nous dressons ensuite l'inventaire des pratiques pédagogiques «exemplaires» identifiées par les enseignants de l'École de bibliothéconomie et des sciences de l'information (EBSI). Enfin, nous tirons quelques constats à partir de la littérature recensée et de l'inventaire effectué.

\section{The Best Pedagogical Practices in Information Sciences}

This article describes the best pedagogical practices in information sciences. Following a discussion of the context and the methodology, the authors present a literature review in three parts: a theoretical discussion of the pedagogical practices in information sciences, a list of suggested teaching methods and a summary of the experiences reported by professors. The authors drafted an inventory of the best pedagogical practices reported by the professors of the École de bibliothéconomie et des sciences de linformation. Finally, they presented a few conclusions based on the literature reviewed and the inventory.

\section{Las "mejores" prácticas pedagógicas en ciencias de la información}

El artículo se interesa en las "mejores" prácticas pedagógicas relacionadas al campo de las ciencias de la información. Luego de contextualizar el tema y formular algunas precisiones de orden metodológico, se presenta una recensión de literatura dividida en tres partes: reflexiones teóricas sobre prácticas pedagógicas en ciencias de la información, guías que proponen enfoques de enseñanza y balances de experiencia informados por profesores. Seguidamente, se elabora el inventario de las "mejores" prácticas pedagógicas identificadas por los profesores de la Escuela de Biblioteconomía y Ciencias de la Información. Por último, se da a conocer algunas conclusiones obtenidas de la recensión de literatura y del inventario realizado.

\section{Mise en contexte}

L es ressources pédagogiques consacrées à l'enseignement des sciences de l'information sont peu nombreuses. Ce constat a émergé d'une recherche documentaire, effectuée entre 2004 et 2006, portant sur les publications existantes, les témoignages d'enseignants et les ressources Web sur les pratiques pédagogiques en sciences de l'information. De ce constat est né un projet de banque de ressources pédagogiques en sciences de l'information, dont le premier volet portait spécifiquement sur l'inventaire des pratiques pédagogiques "exemplaires" (best practices). Cela a permis de dresser l'état de la question sur les pratiques pédagogiques "exemplaires» en sciences de l'information, d'identifier les pratiques les plus utilisées par les professeurs et chargés de cours de l'École de bibliothéconomie et des sciences de l'information (EBSI) de l'Université de Montréal, et d'en décrire un certain nombre en profondeur' ${ }^{1}$.

Le projet vise à mettre à la disposition des enseignants en sciences de l'information une diversité de ressources pédagogiques actuellement dispersées et donc difficilement exploitables. Il vise également le partage des compétences et des expertises des professeurs et chargés de cours en matière d'expériences pédagogiques. L'inventaire des pratiques pédagogiques «exemplaires» a été réalisé dans le cadre des activités du Comité local d'intégration pédagogique (CLIP) de l'EBSI, grâce au soutien financier du Comité universitaire d'intégration pédagogique (CUIP), qui réunit des représentants du Syndicat des chargées et chargés de cours et du Bureau du personnel enseignant de l'Université de Montréal.

Après quelques précisions d'ordre méthodologique, cet article présente tout d'abord un état de la question sur les pratiques pédagogiques en sciences

1 Nous remercions les personnes qui ont collaboré à ce projet: Luce Payette et Sabine Mas, chargées de cours, et Lyne Da Sylva et Éric Leroux, professeurs adjoints, à l'EBSI. 
de l'information. Il dresse ensuite l'inventaire des pratiques considérées comme étant les plus efficaces par les professeurs et les chargés de cours de l'EBSI. Les résultats obtenus grâce à la revue de la littérature et à l'inventaire des pratiques orientent différents constats sur les pratiques pédagogiques « exemplaires» en sciences de l'information.

\section{Méthodologie}

Afin de conserver un caractère réaliste au projet d'inventaire des pratiques pédagogiques « exemplaires » en sciences de l'information ${ }^{2}$, les limites spatiales de la recherche documentaire ont été circonscrites prioritairement au Canada et aux États-Unis, sauf quelques exceptions pour des pays francophones. La recherche documentaire a couvert plusieurs sources d'information, faisant appel tant à une recherche bibliographique dans des bases de données spécialisées qu'à une recherche d'information sur le Web (par exemple: ressources pédagogiques existantes dans les sites Web des universités offrant des programmes en sciences de l'information, dans les sites d'associations professionnelles en sciences de l'information et dans les listes de discussion en sciences de l'information). Un message a été envoyé à six listes de discussion afin de solliciter la collaboration de leurs membres à notre collecte d'information (EBSI-L, Biblio-fr, ARCAN-L, adbs-info, IS-World et JESSE). L'analyse des résultats obtenus à ce stade a d'abord permis d'identifier les principales pratiques pédagogiques utilisées dans l'enseignement, puis d'examiner la perspective adoptée par différents auteurs sur les pratiques pédagogiques en sciences de l'information.

Compte tenu du peu d'information existant sur les pratiques pédagogiques en sciences de l'information, tant dans la littérature que dans les ressources Web, il nous semblait essentiel de recueillir des données de première main sur les pratiques privilégiées par des enseignants de différents programmes d'enseignement dans le domaine. Pour la collecte des données, nous avons tout d'abord sollicité l'ensemble des professeurs de l'EBSI et un échantillon de chargés de cours. La réponse a été très positive puisque nous avons rencontré en entrevue 18 enseignants (14 professeurs et 4 chargés de cours). Pour assurer une collecte de données uniforme lors de ces entrevues en personne, nous avons conçu un questionnaire dont la validité a été testée en effectuant un pré-test avec les deux professeurs collaborant au projet.

Dans un souci de joindre des enseignants d'autres disciplines pour des fins de comparaison, le questionnaire a été envoyé par courriel à des enseignants de

2 La majeure partie de ce projet d'intégration pédagogique a été réalisée durant une session universitaire en 2004. Des mises à jour ont été effectuées en 2005 et en 2006 . domaines connexes $(n=14)$ à l'Université de Montréal: éducation, communication, histoire, histoire de l'art et psychologie. Nous avons obtenu la collaboration du Centre d'études et de formation en enseignement supérieur (CEFES) de l'Université de Montréal pour l'identification de ces enseignants. Malheureusement, nous n'avons reçu aucune réponse de ces derniers.

\section{Recension des écrits}

Les principales perspectives identifiées dans les écrits sur les pratiques pédagogiques en sciences de l'information peuvent être subdivisées en trois catégories:

a) Des réflexions théoriques sur les pratiques pédagogiques en sciences de l'information, en général ;

b) Des guides proposant des approches d'enseignement ;

c) Des bilans d'expériences rapportés par des enseignants ayant expérimenté une pratique pédagogique en particulier dans l'enseignement d'une matière donnée.

\section{Réflexions théoriques sur les pratiques pédagogiques en sciences de l'information}

Datant de la fin des années 1960, l'ouvrage de J.E. Sabor (1969) est exclusivement consacré aux méthodes d'enseignement de la bibliothéconomie. L'auteur souligne d'emblée que cette discipline n'a pas mis au point une méthode didactique qui lui soit propre, mais a plutôt emprunté des méthodes d'autres disciplines telles que le droit ou l'économie (par exemple, l'étude de cas). L'auteur passe en revue 19 méthodes d'enseignement réparties en quatre regroupements :

a) La méthode de l'exposé;

b) La démonstration, le commentaire, l'interrogation, la conversation et la discussion;

c) De l'observation à la recherche; et

d) La recherche et les travaux écrits.

L'utilisation "d'auxiliaires d'enseignement» comme matériel didactique est mise en relief, ces auxiliaires pouvant être du matériel audiovisuel (films, diapositives, etc.) ou encore des illustrations (services, bâtiments, mobilier, etc.).

J.E. Sabor (1969) rappelle l'interrelation qui existe entre l'enseignement de la bibliothéconomie et les besoins auxquels doit répondre la bibliothèque dans son milieu, cette interrelation étant à l'origine des variations que connaît constamment la discipline:

«La bibliothéconomie est une discipline dont le contenu varie constamment, non seulement 
dans la mesure où la matière bibliothéconomique elle-même devient plus riche et plus complexe, mais encore parce que les objectifs d'enseignement changent selon les variations $d u$ milieu social auquel la bibliothèque fournit ses services et les exigences nouvelles de ce milieu.» (Sabor, 1969: 7o)

En se basant sur les opinions et les recommandations des professeurs des écoles de bibliothécaires, J.E. Sabor (1969) dresse une liste des méthodes les plus indiquées pour l'enseignement des matières constituant la base de la bibliothéconomie (par exemple: Histoire du livre et des bibliothèques, Référence et bibliographie, Catalogage et classification, etc.). L'ouvrage s'achève avec une bibliographie «fondamentale» traitant de la formation de bibliothécaires en général, qui serait utile pour des études s'intéressant à l'historique et à l'évolution de l'enseignement de la bibliothéconomie au $\mathrm{xx}^{\mathrm{e}}$ siècle.

Le colloque "Théorie et pratique dans l'enseignement des sciences de l'information" (Savard, 1988) - qui s'est tenu à Montréal et était organisé conjointement par l'Association internationale des écoles en sciences de l'information (AIESI) et l'Association for Library and Information Science Education (ALISE) - avait pour but d'examiner l'équilibre à conserver entre théorie et pratique dans les programmes en sciences de l'information. Pour ce faire, trois thématiques ont été débattues en plénières:

1. 1) le «Contexte» a permis de traiter des programmes de formation, de l'enseignement de l'archivistique au sein des sciences de l'information, de la recherche en enseignement et du rôle des approches pédagogiques en sciences de l'information;

2. 2) le «Débat» a mis l'accent sur l'enseignement de matières spécifiques (notamment le traitement de l'information et l'analyse de systèmes) et sur l'apport des nouvelles technologies à la formation en sciences de l'information; et

3. 3) le «Futur» a permis d'aborder des sujets comme les acteurs de la formation en sciences de l'information, la formation à l'interrogation des banques de données, la formation continue et la formation en sciences de l'information dans d'autres pays.

Ce colloque a aussi été l'occasion de présenter des expériences menées dans des pays en développement (au Gabon, en Albanie et au Sénégal) et des pays francophones d'Europe (à Toulouse, à Aix-Marseille et en Belgique). Quelques développements dans les pays arabes (cas du Maroc et de l'Algérie) ont été également exposés.

En 1998, la revue Journal of Education for Library and Information Science consacrait un numéro à l'enseignement de la bibliothéconomie et des sciences de l'information. D. M. Shannon (1998) s'y intéresse aux comportements d'enseignement efficaces chez les professeurs de niveau post-secondaire en général et chez les professeurs en bibliothéconomie et sciences de l'information en particulier. Faisant une synthèse de ce qu'en dit la littérature, l'auteure dégage les caractéristiques permettant d'évaluer en quoi consiste un excellent professeur, qui peuvent être, soit basées sur le jugement des étudiants (par exemple, flexibilité, enthousiasme, clarté du propos), soit observées avec une plus grande part d'objectivité (par exemple, le professeur donne plusieurs exemples, il bouge pendant qu'il donne son cours, etc.). Ces caractéristiques ont été utilisées dans des études sur la relation "processproduct $^{3}$ ", dans des études sur les perceptions des étudiants et des professeurs sur ce que constitue un enseignement efficace, et dans des études qui mettent en évidence l'importance des relations verbales et non verbales entre étudiants et professeurs. D.M. Shannon présente ensuite trois modèles proposés dans la littérature pour favoriser la qualité de l'enseignement: 1) Two-Dimensional Model; 2) Seven Principles for Good Practice; et 3) A Five-Step Process for Improving Teaching. Chaque modèle comporte des techniques et des stratégies qui font office de recommandations pour aider les professeurs à évaluer leur enseignement et à susciter la discussion avec leurs pairs. Lauteure termine son article en précisant que la recherche sur les comportements d'enseignement reste encore un territoire à explorer.

C. Curran (1998), pour sa part, présente les résultats d'une enquête menée auprès de 61 professeurs en sciences de l'information et visant à examiner comment ils planifient, comment ils enseignent et comment ils interagissent avec leurs étudiants. Ces professeurs avaient été identifiés comme étant des «superior teachers» par des directeurs de départements d'universités américaines et canadiennes. Les thèmes traités par les professeurs au cours de cette enquête ont été réunis en trois volets: 1) planification et préparation des cours; 2) prestation des cours; et 3) considérations affectives. Par ailleurs, C. Curran a complété l'enquête auprès des professeurs en recueillant les propos tenus par six directeurs de départements sur un forum de discussion. Ces échanges ont permis d'obtenir leur point de vue sur un enseignement de haute qualité, sur leurs critères pour qualifier un professeur de "superior teacher», sur la possibilité d'améliorer la qualité de l'enseignement, et enfin sur les considérations dont il faut tenir compte dans l'enseignement aux adultes en sciences de l'information. Au-delà de toutes les réponses fournies par les professeurs et les directeurs de départements, il est apparu que plusieurs d'entre eux ont identifié l'écoute comme une "compétence supérieure» en enseignement en sciences de l'information.

3 Où «process» correspond au fait d'enseigner et «product», au résultat produit chez l'étudiant, qu'il s'agisse d'acquis cognitifs ou affectifs. 
Enfin, D. Carr (1998) fonde son article sur le fait que l'acte d'enseigner se produit souvent dans des conditions de tension. L'auteur souhaite décrire quelques-unes de ces tensions et les effets qu'elles peuvent avoir sur les expériences d'enseignement en salle de cours. Parmi celles qu'il aborde, mentionnons les tensions créées par les valeurs du professeur ${ }^{4}$, les tensions créées par la définition donnée par le professeur au concept de «connaissances ${ }^{5}$ », les tensions créées par le manque de véritables évaluations critiques et formatives, ou encore les tensions créées par la reconnaissance de l'excellence de l'enseignement ${ }^{6}$. L'auteur termine par une série de recommandations destinées à contrer les pièges inhérents à ces tensions.

\section{Guides pratiques proposant des approches d'enseignement}

Parmi les écrits recensés, nous avons identifié des ouvrages se présentant comme des guides pratiques. En 1982, M. Cook rédigeait des «Principes directeurs pour l'élaboration de programmes d'enseignement dans le domaine de la gestion des documents et de l'administration des archives modernes» pour le compte de l'Unesco. Il y traite notamment des objectifs poursuivis par différentes méthodes pédagogiques: les cours magistraux, les séminaires, les exercices pratiques, les «études spéciales», au sens d'«études pratiques approfondies», qui seraient comparables à des mémoires et permettraient d'étudier des problématiques spécifiques. Il aborde d'autres méthodes pédagogiques en mettant l'accent, cette fois, sur les problèmes qu'elles peuvent soulever. Parmi ces méthodes, mentionnons les visites professionnelles dans des services d'information établis et les démonstrations.

Quelques années plus tard, dans un guide conçu dans le cadre d'un accord entre l'Unesco et le service pédagogique de l'Université de Montréal, F. Fontaine et P. Bernhard (1988) se sont adressées aux enseignants en bibliothéconomie, en sciences de l'information et en archivistique pour faciliter la rédaction des objectifs d'apprentissage en ces domaines, et ce, au moyen d'exercices pratiques et d'explications. Les auteures soulignent dans la préface que:

"Dans la mesure du possible, la formation des archivistes, des bibliothécaires et des spécialistes de l'information doit être conçue de manière concertée par les différentes instan-

4 Les valeurs du professeur peuvent être différentes des valeurs prônées par l'université en matière d'enseignement.

5 L'avancement des connaissances dans une salle de cours ne correspond pas nécessairement à ce que l'université entend par l'avancement des connaissances grâce à la recherche.

6 Un bon professeur se voit souvent récompensé par des classes plus nombreuses, par la réputation d'être attentif aux besoins de ses étudiants, et parce qu'il consacre moins de temps à la recherche. ces qui en ont la charge.» (Fontaine et Bern-

hard, 1988: ii)

L'avènement des outils technologiques et des ressources informationnelles numériques a favorisé le développement de nouvelles matières et méthodes d'enseignement en sciences de l'information. Ainsi, au Royaume-Uni, 10 institutions ont participé à un projet qui étudiait l'enseignement de la recherche en ligne (automatisée) dans les bases de données des services commerciaux tels que DIALOG et MEDLARS. B.C. Vickery (1977) présente dans son rapport le résumé des projets conduits par plusieurs écoles. Pour améliorer l'enseignement de la recherche automatisée, l'auteur formule des recommandations en ce qui a trait, entre autres, au nombre d'étudiants par groupe, à l'élaboration et à l'utilisation des outils d'aide à l'enseignement (démonstrations, didacticiels, etc.), au choix des formateurs et aux méthodes d'évaluation.

Pour ce qui est de l'apprentissage et de la formation à distance ou en ligne (e-Learning ou Online Learning), B. Allan (2002) fait le tour de la question en présentant: 1) les outils et les technologies d'apprentissage en ligne (par exemple: forums de discussion, listes d'envoi, clavardage, vidéoconférence); 2) l'apprentissage et l'enseignement en ligne; et 3) le lien entre la formation à distance et la profession des sciences de l'information. Dans le chapitre 9, B. Allan dresse un tableau de 12 activités de formation à distance. Ainsi, pour chaque activité sont déclinés ses caractéristiques, les raisons de son utilisation, ses avantages et ses problèmes potentiels. Comme exemples d'activités, mentionnons le brainstorming, les études de cas, les groupes de discussion, les visites virtuelles et les conférenciers virtuels.

\section{Bilans d'expérience}

Plusieurs articles ont été consacrés à des expériences précises menées dans des écoles de sciences de l'information et liées à un aspect particulier de la formation des futurs professionnels. Ainsi en est-il de certains des textes colligés dans les actes du colloque conjoint AIESI-ALISE (Savard, 1988). Parmi les communications présentées à ce colloque, certaines ont traité de méthodes pédagogiques spécifiques éprouvées par les professeurs dans leurs cours:

a) Le séminaire (Tague, 1988): l'évolution de la méthode du séminaire, ses caractéristiques, ses avantages et inconvénients;

b) Les exercices et la méthode des cas (BertrandGastaldy et Brind'amour, 1988): utilité dans l'enseignement de l'analyse documentaire;

c) Les exercices, les mises en situation et les démonstrations (Deschatelets, 1988): principes pédagogiques et outils didactiques pour l'enseignement de linterrogation des banques de données; 
d) Le stage (Ibn Lkhayat, 1988; Yacine, 1988): objectifs, gestion, supervision et évaluation.

M. Sow (2002) explique, quant à lui, comment l'intégration des technologies (notamment les cédéroms et Internet) a changé l'enseignement de la bibliographie pour les bibliothécaires de référence à l'École des bibliothécaires, archivistes et documentalistes de Dakar. A.S. Warner (1999), de son côté, a développé un cours sur l'enseignement de la gestion fiscale et budgétaire des bibliothèques à la Simmons Graduate School of Library and Information Science, à Boston (Massachusetts). Outre la description du cours, elle expose dans son article les méthodes pédagogiques qu'elle utilise. A. Slaviae (2001) s'attarde à l'enseignement de la classification en Croatie, à l'Université de Zagreb. En prenant pour exemple le cours "Classification et systèmes de classification", elle explique l'évolution qu'a connue l'enseignement de cette matière qui s'appuie, entre autres, sur l'usage d'Internet en l'absence de réseau de ressources bibliothéconomiques croates. D'autres sujets ont fait l'objet d'expériences tentées par des professeurs, par exemple l'utilisation des formats disponibles (notamment ceux du World Wide Web Consortium) dans l'enseignement du multimédia (Turner, 2003), l'enseignement du catalogage à distance avec le système WebBoard (Harcourt et Neumeister, 2002), ou encore le portfolio, la cartographie conceptuelle («mindmapping») et l'évaluation des pairs dans un cours d'introduction aux sciences de l'information (Kortelainen et Vanhala, 2004).

La formule du stage suscite la réflexion sur différents aspects spécifiques, par exemple sur le processus d'apprentissage chez des étudiants du $1^{\text {er }}$ cycle en sciences de l'information (Brown et Murphy, 2005), ou encore sur l'utilisation d'un forum Internet pour permettre à des stagiaires de maintenir la communication entre eux (Macchia et Freedman, 2004). L'apport du stage à la formation professionnelle initiale des archivistes au Québec, de 1983 à 1994, a fait l'objet d'une thèse de doctorat (Rousseau, 1997). Cette recherche est fondée à la fois sur l'expérience professionnelle de l'auteur, sur une revue de la littérature, sur des rencontres avec des coordonnateurs de stage en archivistique d'universités québécoises et, enfin, sur une enquête menée auprès de diplômés en archivistique qui étaient alors membres d'une association professionnelle ayant des activités au Québec et qui travaillaient dans le domaine des archives. Parmi ses conclusions, l'auteur affirme que le stage favorise davantage l'acquisition de connaissances pratiques que théoriques, facilite l'intégration de la théorie à la pratique et contribue au développement d'un regard critique sur la profession. Toutefois, il précise que le stage ne suffit pas à acquérir une autonomie professionnelle ni la maitrise de l'acte professionnel. L'auteur discute en outre de l'utilité d'autres activités pratiques dans la formation professionnelle initiale des archivistes, notamment l'analyse de procédure, l'analyse de système, l'étude de cas, la visite en milieu professionnel et le travail en laboratoire.

Par ailleurs, trois professeurs du département d'Information Science de la Loughborough University ont élaboré une revue de la littérature portant sur les méthodes et les techniques d'enseignement en sciences de l'information expérimentées au Royaume-Uni (Odhiambo, Stephens et Goulding, 2002). Cette revue de la littérature couvre une période de 10 ans (à partir de l'année 1990) et est circonscrite aux périodiques académiques recensés dans les bases de données ERIC (Education Resources Information Center) et LISA (Library and Information Science Abstracts). De cette manière, les auteurs espèrent identifier ce qui constitue une «bonne pratique» dans l'enseignement en sciences de l'information. Ils précisent d'entrée de jeu qu'ils ne s'intéressent pas, dans le cadre de leur article, aux cours magistraux, mais plutôt aux autres méthodes d'enseignement. Ils regroupent en deux catégories les articles trouvés dans les bases de données. La première catégorie porte sur des textes mettant l'accent sur la manière dont différents médias sont utilisés dans l'enseignement des sciences de l'information. Il y est notamment question de l'utilisation de la vidéoconférence, et de l'enseignement à l'aide de l'informatique et des ressources de l'Internet. La seconde catégorie inclut des textes portant sur des techniques utilisées pour enseigner le «contenu» des sciences de l'information. Les auteurs traitent alors de l'apprentissage en groupes pour développer des compétences en communication; de l'étude de cas pour expérimenter la réalité et la complexité du monde du travail; du jeu qui s'inscrit dans la logique du «learning by doing»; du stage; des exercices en laboratoire d'informatique, et des ateliers restreints pour développer des compétences pratiques sous supervision. F. Odhiambo, D. Stephens et A. Goulding terminent l'article en présentant leurs prédictions sur ce que sera l'enseignement des sciences de linformation en 2011. Ils croient notamment qu'il y aurait avantage à emprunter des méthodes issues de l'approche technologique de l'enseignement à distance et de les intégrer aux cours donnés sur le campus. Ils espèrent que la bonification de l'infrastructure et des ressources (support technique et expertise) permettra aux professeurs de bénéficier de tout le potentiel technologique pour la présentation de leurs cours. Ils souhaitent aussi le développement d'une approche plus collaborative relativement au matériel et aux méthodes d'enseignement et d'évaluation, ce qui permettrait de mieux faire face aux différentes problématiques soulevées dans leur article. 


\section{Inventaire des pratiques pédagogiques}

$\mathrm{Au}$ cours des entrevues avec les enseignants en sciences de l'information, nous leur avons demandé de nous indiquer les pratiques pédagogiques qu'ils utilisent le plus fréquemment dans leurs cours. Parmi ces pratiques, nous leur avons ensuite demandé d'en choisir quatre qu'ils considèrent comme étant les meilleures en termes d'utilité et d'efficacité: ces pratiques ont été décrites en profondeur à l'aide d'une grille assurant l'homogénéité de la description d'un enseignant à l'autre. Par ailleurs, une grille d'analyse des pratiques pédagogiques exemplaires a été établie, de manière à faciliter tant la collecte de données que leur analyse. Cette grille d'analyse, présentée en annexe 1, comporte les éléments suivants:

1. titre de la pratique pédagogique;

2. termes rejetés;

3. description de la pratique;

4. contexte d'utilisation;

5. notes d'application;

6. évaluation de l'apprentissage fait à l'aide de la pratique;

7. évaluation de la pratique par les étudiants et par les enseignants;

8. recommandations, au besoin;

9. lien avec d'autres méthodes pédagogiques, au besoin.

Une liste synthétique de toutes les pratiques pédagogiques mentionnées par les enseignants a été dressée pour obtenir un portrait complet de l'ensemble des pratiques utilisées (annexe 2). Une autre liste distincte a été établie pour les pratiques pédagogiques décrites en profondeur par les enseignants, où nous avons effectué un regroupement des pratiques similaires. Nous nous sommes basées pour ce faire sur les descriptions fournies par les enseignants, de même que sur des ouvrages en éducation sur le sujet. À partir de cette seconde liste, 21 pratiques ont été retenues et ont fait l'objet de fiches annotées. Leur choix a été fondé sur différents critères, dont la représentativité (les pratiques mentionnées le plus souvent, comme le compte rendu de lecture, les conférenciers invités, les exercices en classe, l'exposé magistral, les travaux pratiques, ou la visite de milieu), mais également le caractère unique et original de certaines (par exemple, les questions populaires ou la recherche commanditée). Les membres du projet ont aussi essayé de retenir des pratiques pédagogiques utilisées pour des cours de nature tant théorique que pratique, compte tenu du caractère professionnel de l'enseignement donné à l'EBSI.

Les 21 fiches annotées portent sur les pratiques pédagogiques suivantes, présentées selon l'ordre alphabétique de leur intitulé:
1. Abonnement à des listes de discussion;

2. Activité de formation des utilisateurs;

3. Apprentissage par projet;

4. Compte rendu de lecture;

5. Conférencier invité;

6. Constitution de dossiers sur les organisations;

7. Construction de ressources Web;

8. Discussion/débat;

9. Étude de cas;

10. Exercice en classe;

11. Exposé magistral;

12. Lecture;

13. Présentation des étudiants devant public;

14. Questions populaires;

15. Jeu questionnaire;

16. Rapport d'étonnement;

17. Recherche d'information commanditée;

18. Table ronde;

19. Travail pratique;

20. Travail sur l'actualité;

21. Visite de milieu.

Deux fiches détaillées de deux pratiques pédagogiques sont présentées, à titre d'exemples, en annexe 3 . La première pratique, "Questions populaires», a été choisie pour son caractère unique (une seule chargée de cours l'utilisait au moment de l'enquête). La seconde, "Visite de milieu», était utilisée dans quatre cours au moment de l'enquête, son application étant spécifique aux objectifs de chaque cours.

La majorité des 21 pratiques pédagogiques décrites en profondeur par les enseignants sont issues des pratiques que l'on retrouve généralement en éducation. Leur contenu est adapté aux volets théoriques (par exemple: exposés magistraux, comptes rendus de lectures, présentations des étudiants devant public) et aux volets pratiques (par exemple: exercices en classe, études de cas, apprentissage par projet, visites de milieux) de l'apprentissage en sciences de l'information. Certaines pratiques pédagogiques sont plus spécifiques aux sciences de l'information, par exemple, l'activité de formation des utilisateurs, la constitution de dossiers sur les organisations, la construction de ressources Web et la recherche d'information commanditée. Sur 21 pratiques pédagogiques, 13 servent de moyens d'évaluation de l'apprentissage des étudiants et 6 ne sont pas notées. Dans le cas de ces dernières (par exemple: exposés magistraux, lectures, conférenciers invités), la matière qui est abordée est évaluée à l'aide d'autres moyens comme les examens. Deux pratiques (exercices en classe et travaux pratiques) servent de moyens d'évaluation dans certains cours mais pas dans d'autres. 


\section{Constats sur les pratiques pédagogiques " exemplaires» en sciences de l'information}

Sans prétendre être exhaustive, la recension des écrits témoigne du manque d'ouvrages actualisés traitant des pratiques pédagogiques en sciences de l'information. Bien que les réflexions sur l'enseignement de la discipline en général ne manquent pas, la recherche documentaire a permis de constater que plusieurs écrits pertinents se sont surtout concentrés sur l'enseignement d'une seule matière ou l'application d'une seule pratique pédagogique. D.M. Shannon (1998) observe qu'il existe peu de recherche empirique, en sciences de l'information, qui porte spécifiquement sur les comportements d'enseignement. Elle considère qu'il y a surtout des publications sur les programmes d'enseignement, sur l'impact des technologies sur l'enseignement des sciences de l'information et sur les pratiques d'enseignement. F. Odhiambo, D. Stephens et A. Goulding (2002) affirment avoir repéré moins d'articles que ce qu'ils anticipaient, pour la période de 1990 à 2000, dans les revues de sciences de l'information avec comités des pairs recensées par les bases de données ERIC et LISA. Le nombre d'articles qu'ils ont trouvés sur les pratiques d'enseignement s'avère, selon eux, très faible comparativement au nombre d'articles sur la structure et le contenu des programmes d'enseignement. Les auteurs expliquent cela par le fait que les publications sur l'enseignement ne seraient pas prises en considération dans l'évaluation des activités de recherche des professeurs en sciences de l'information, au Royaume-Uni en ce qui les concerne.

La recherche documentaire effectuée dès le début du projet révèle que les sources d'information les plus riches sur les pratiques pédagogiques ne proviennent pas du domaine des sciences de l'information. Les ressources trouvées en sciences de l'éducation offrent un portrait assez complet et détaillé des pratiques pédagogiques existantes, de leurs forces et de leurs limites, et de leurs modalités d'application (lesquelles prennent parfois la forme de consignes très détaillées, tant pour la part de l'enseignant que pour celle de l'étudiant). Citons, dans cet ordre d'idées, l'ouvrage de G. Chamberland, L. Lavoie et D. Marquis (200o) sur les formules pédagogiques et celui de M. Bédard, P. Dell'Aniello et D. Desbiens (1991) sur la méthode des cas.

Il ressort également qu'il est difficile de qualifier une pratique pédagogique d'«exemplaire». Le succès de chaque pratique pédagogique, en effet, réside dans sa pertinence à répondre aux objectifs du cours. Cela dépend à la fois du sujet traité dans le cours et de la nature du cours (théorique ou pratique). C'est aussi ce qui émerge de l'enquête menée auprès des enseignants de l'EBSI, où l'expression «pratique pédagogique exemplaire » a suscité des interrogations. Les enseignants, en effet, ont hésité à qualifier leurs pratiques d'exemplaires, tout en précisant bien que chaque pratique ne prend tout son sens que dans le contexte du cours et des objectifs spécifiques de ce cours. Parmi toutes les pratiques pédagogiques qu'ils utilisent, les enseignants ont choisi eux-mêmes les quatre pratiques qu'ils souhaitaient décrire en profondeur. Ces pratiques étaient celles qu'ils considéraient les plus utilisées, les plus efficaces ou les plus originales. Par conséquent, il s'avère impossible de déterminer si une pratique pédagogique peut être "meilleure» qu'une autre. Il est toutefois possible d'affirmer qu'une pratique est «exemplaire» lorsqu'elle fonctionne bien dans un cours, au regard des objectifs pédagogiques de ce cours.

\section{Conclusion}

La recension des écrits a permis de constater qu'il reste encore de la place à l'examen des différentes pratiques pédagogiques en sciences de l'information, de même quà l'examen de la perception de leurs avantages et inconvénients par les enseignants de la discipline. Le succès rencontré au moment de la diffusion des résultats 7 du présent projet auprès des enseignants de l'EBSI et d'autres enseignants francophones en sciences de l'information (notamment en France et en Suisse) favorise de futurs développements dans le but d'augmenter le nombre de fiches annotées sur les pratiques pédagogiques en sciences de l'information, et d'explorer de nouvelles pratiques peu connues dans le domaine, généralement empruntées d'autres disciplines (par exemple, la pédagogie par projet).

Par ailleurs, au cours de notre enquête auprès des enseignants de l'EBSI, nous avons constaté une forte volonté de communiquer et de partager les savoirs et les savoir-faire. En quelque sorte, il existe une communauté de pratiques qui demande à s'exprimer par davantage de moyens qu'auparavant (par exemple, les réunions périodiques de chargés de cours des différents programmes). L'inventaire des pratiques pédagogiques constitue un des moyens de mettre en commun des ressources à caractère pédagogique en sciences de l'information. D'autres moyens ont également été mis en place au cours des dernières années, dont une terminologie de base en sciences de l'information. Ces ressources sont accessibles aux professeurs et aux chargés de cours sur un portail (<http://www.ebsi. umontreal.ca/clip/ >) dont le contenu évoluera avec les années. (-)

\footnotetext{
7 Les résultats du projet ont été diffusés sur les principales listes de discussion en sciences de l'information (EBSI-L, Biblio-fr, ARCAN-L, adbs-info, IS-World et JESSE) et par le dépôt de ces résultats dans des sites d'archives ouvertes en sciences de l'information et de la communication: Archivesic (<http://archivesic. ccsd.cnrs.fr/ >) et Papyrus (<https://papyrus.bib.umontreal.ca/dspace/>).
} 


\section{Sources consultées}

Allan, B. 2002. E-Learning and Teaching in Library and Information Services. London, Facet. xiv-273 p.

Bédard, M., P. Dell'Aniello et D. Desbiens. 1991. La Méthode des cas: Guide d'analyse, d'enseignement et de rédaction. Montréal, Gaëtan Morin éditeur, xiii-9o p.

Bertrand-Gastaldy, S. et N. Brind'amour. 1988. Pratique et théorie dans l'enseignement de l'analyse documentaire. [résumé] In R. Savard (dir.), Théorie et pratique dans l'enseignement des sciences de l'information: Comptes rendus du premier Colloque conjoint entre l'Association internationale des Écoles de sciences de l'information (AIESI) et l'Association for Library and Information Science Education (ALISE), Montréal, Canada, 25-27 mai 1988. [Montréal], École de bibliothéconomie et des sciences de l'information, Université de Montréal, p. 297.

Brown, C.M., T.J. Murphy. 2005. Understanding student learning in undergraduate information studies internships. Journal of Education for Library and Information Science, 46 (3): 234-247.

Carr, D. 1998. Tensions of Teaching. Journal of Education for Library and Information Science, 39 (3): 195-203.

Chamberland, G., L. Lavoie et D. Marquis. 2000. 20 formules pédagogiques. Sainte-Foy, Presses de l'Université du Québec, xvi-176 p. (Formules pédagogiques.)

Cook, M. 1982. Principes directeurs pour l'élaboration de programmes d'enseignement dans le domaine de la gestion des documents et de l'administration des archives modernes: Une étude du RAMP. Paris, UNESCO. 70 p. (PGI-82/WS/16).

Curran, C. 1998. What sixty-one Superior LIS Teachers say about Superior LIS Teaching, Plus Comments from Six Knowledgeable Observers. Journal of Education for Library and Information Science, 39 (3): 183-194.

Deschatelets, G. 1988. Méthodes d'enseignement et outils d'aide à la formation à la recherche documentaire automatisée. In R. Savard (dir.), Théorie et pratique dans l'enseignement des sciences de l'information: Comptes rendus du premier Colloque conjoint entre l'Association internationale des Écoles de sciences de l'information (AIESI) et l'Association for Library and Information Science Education (ALISE), Montréal, Canada, 25-27 mai 1988. [Montréal], École de bibliothéconomie et des sciences de l'information, Université de Montréal, p. 429-465.

Fontaine, F. et P. Bernhard. 1988. Principes directeurs pour la rédaction d'objectifs d'apprentissage en bibliothéconomie, en sciences de l'information et en archivistique. Programme général d'information. UNISIST. Paris, Unesco, $128 \mathrm{p}$

Harcourt, K. et S.M. Neumeister. 2002. Online distance learning with cataloging mentors: The mentor's viewpoint. Cataloging and Classification Quarterly, 34 (3): 293-298.

Ibn Lkhayat, N. 1988. Le stage, élément de formation en sciences de l'information dans les pays périphériques: cas du Maroc. In R. Savard (dir.), Théorie et pratique dans l'enseignement des sciences de l'information: Comptes rendus du premier Colloque conjoint entre l'Association internationale des É coles de sciences de l'information (AIESI) et l'Association for Library and Information Science Education (ALISE), Montréal, Canada, 25-27 mai 1988. [Montréal], École de bibliothéconomie et des sciences de l'information, Université de Montréal, p. 511-523.

Kortelainen, T.; Vanhala, M. 2004. Portfolio, peer evaluation, and mind map in an introductory course of information studies. Journal of Education for Library and Information Science, 45 (4) : 273-285.

Macchia, P. Jr. et J.S. Freedman. 2004. From isolation to communication: The use of Internet forums to build a learning community among library interns. Journal of Education for Library and Information Science, 45 (3): 200-209.
Odhiambo, F., D. Stephens et A. Goulding. 2002. Teaching and assessment methods in UK information science: A ten year review of professional and scholarly journal content and predictions for 2011. Education for Information, 20:183-198.

Rousseau, J.-Y. 1997. Étude descriptive de la contribution du stage à la formation professionnelle initiale des archivistes au Québec de 1983 à 1994. Thèse de doctorat. Montréal, Département de didactique, Université de Montréal, 296 p.

Sabor, J.E.; Ricardo, N. 1969. Méthodes d'enseignement de la bibliothéconomie. Paris, Unesco, 154 p.

Savard, R. (dir.). 1988. Théorie et pratique dans l'enseignement des sciences de l'information: Comptes rendus du premier Colloque conjoint entre l'Association internationale des Écoles de sciences de l'information (AIESI) et l'Association for Library and Information Science Education (ALISE), Montréal, Canada, 25-27 mai 1988. [Montréal], École de bibliothéconomie et des sciences de l'information, Université de Montréal. xiii-538 p.

Shannon, D. M. 1998. Effective teacher behaviors in higher education and in LIS education programs: A review of the literature. Journal of Education for Library and Information Science, 39 (3): 163-174.

Slaviae, A. 2001. L'enseignement de la classification au sein d'un cursus moderne et stable en bibliothéconomie et sciences de l'information: l'exemple de la Croatie. $67^{e}$ Congrès de l'IFLA. Boston, États-Unis, 16-25 août 2001. Adresse URL: < http:// www.ifla.org/IV/ifla67/papers/o3o-14.2f.pdf > (page consultée le 28 juillet 2005).

Sow, M. 2002. La formation des bibliothécaires de référence à l'École des bibliothécaires, archivistes et documentalistes de l'Université Cheikh Anta Diop de Dakar. $68^{e}$ Congrès de l'IFLA Glasgow, Écosse, 18-24 août 2002. Adresse URL: < http://www. ifla.org/IV/ifla68/papers/oo3-128f.pdf > (page consultée le 28 juillet 2005).

Tague, J. 1988. The evolution of the seminar method. In R. Savard (dir.), Théorie et pratique dans l'enseignement des sciences de l'information: Comptes rendus du premier Colloque conjoint entre l'Association internationale des Écoles de sciences de l'information (AIESI) et l'Association for Library and Information Science Education (ALISE), Montréal, Canada, 25-27 mai 1988. [Montréal], École de bibliothéconomie et des sciences de l'information, Université de Montréal, p. 185-194.

Turner, J.M. 2003. Teaching the use of publicly-available formats for multimedia as part of a library school curriculum: The vision and the reality. $69^{e}$ Congrès de l'IFLA. Berlin, Allemagne, 1-9 août 2003. Adresse URL: < http://www.ifla.org/IV/ifla69/ papers/o37e-Turner.pdf $>$ (page consultée le 28 juillet 2005).

Vickery, B.C. 1977. The Use of On-Line Search in Teaching: An Assessment of Projects Carried Out by UK Schools of Library and Information Studies. London, British Library. 43 p.

Warner, A.S. 1999. Teaching about library budgets. The Bottom Line: Managing Library Finances, 12 (1): 19-21.

Yacine, B. 1988. L'enseignement théorique et pratique de la bibliothéconomie à l'Institut de bibliothéconomie et sciences documentaires d'Oran. In R. Savard (dir.), Théorie et pratique dans l'enseignement des sciences de l'information: Comptes rendus du premier Colloque conjoint entre l'Association internationale des Écoles de sciences de l'information (AIESI) et $l$ 'Association for Library and Information Science Education (ALISE), Montréal, Canada, 25-27 mai 1988. [Montréal], École de bibliothéconomie et des sciences de l'information, Université de Montréal, p. 525-538. 


\section{Annexes}

\section{Annexe 1. Structure des fiches annotées sur les pratiques pédagogiques des enseignants de l'EBSI}

1. Titre de la pratique pédagogique: De manière générale, le singulier est utilisé pour désigner la pratique pédagogique.

2. Employé pour: Indique les termes utilisés par les enseignants pour des pratiques pédagogiques semblables qui ont été regroupées.

3. Description: Comporte une description générale de la pratique pédagogique tel que cela se retrouve dans les explications fournies par les enseignants, de même que les particularités de la pratique selon les cours où elle est mise en application. Pour certaines pratiques, on retrouve aussi une description tirée de la littérature en éducation lorsque cela peut apporter des éclaircissements utiles.

4. Contexte d'utilisation (échantillon de l'enquête): Correspond aux cours où la pratique pédagogique est mise en application. À des fins de référence ultérieure, le contexte est indiqué par le sigle et le titre du cours, le nom du programme dans lequel il s'inscrit et le nom de l'enseignant qui le donnait au moment de l'enquête sur les pratiques pédagogiques.

Dans les cours obligatoires, on trouve généralement un grand nombre d'étudiants. Quant aux cours optionnels de $2^{\mathrm{e}}$ année de maîtrise, le nombre d'étudiants peut être plus restreint et favoriser ainsi des formules pédagogiques différentes (par exemple, l'apprentissage par projet ou la construction de ressources Web).

Au moment de l'enquête, dans les cours de maîtrise en sciences de l'information, on comptait en moyenne le nombre d'étudiants suivants:

$\triangleright$ Environ 80 étudiants dans les cours obligatoires de $1^{\text {re }}$ année.

$\triangleright$ Environ 80 étudiants dans les cours obligatoires de $2^{\mathrm{e}}$ année.

$\triangleright$ Environ 10 à 20 étudiants dans les cours optionnels de $2^{\mathrm{e}}$ année, quoique dans certains de ces cours, il peut n'y avoir que 5 étudiants.

$\mathrm{Au}$ moment de l'enquête, dans les cours du certificat en archivistique et du certificat en gestion de l'information numérique, on comptait en moyenne le nombre d'étudiants suivants:

$\triangleright$ Environ 50 à 60 étudiants dans les cours obligatoires.

$\triangleright$ Environ 30 étudiants dans les cours optionnels.

5. Application: Fournit des indications concernant la taille de l'équipe lorsque la pratique pédago- gique demande la constitution d'équipes, le temps de réalisation, le lieu de réalisation, le nombre de superviseurs ou d'assistants, le cas échéant, et la fréquence d'utilisation de la pratique dans le cours.

6. Évaluation de l'apprentissage: Indique si les étudiants sont évalués pour l'apprentissage qu'ils font par l'intermédiaire de la pratique et, le cas échéant, le pourcentage attribué à cette évaluation. Des renseignements additionnels sont également fournis sur les personnes chargées de la correction et sur l'existence d'une grille de correction.

7. Évaluation de la pratique: Indique si la pratique pédagogique est évaluée par les étudiants et, si oui, de quelle manière. Indique aussi les avantages et les limites que voit l'enseignant à cette pratique.

8. Recommandations: Fournit les recommandations de l'enseignant quant à la mise en application de cette pratique pédagogique.

9. Liens avec d'autres pratiques pédagogiques: Indique quelles autres pratiques pédagogiques sont utilisées en complémentarité avec la pratique qui est décrite dans la fiche.

\section{Annexe 2. Liste synthétique des pratiques pédagogiques mentionnées par les enseignants de l'EBSI en entrevue}

Note: Cette liste inclut des regroupements de pratiques pédagogiques semblables malgré des appellations différentes.

1. Abonnement obligatoire à deux listes de discussion dans le domaine et compte rendu.

2. Apprentissage par projet.

Employé pour: étude sur le terrain; interventions ponctuelles en milieu de travail; pédagogie par projet.

3. Banque de questions de référence.

4. Compte rendu de lecture.

Employé pour: élaboration du schéma de concepts d'une lecture obligatoire; évaluation de lecture; rapport de lecture; résumé critique; résumé de lecture.

5. Conférenciers invités.

6. Constitution de dossiers sur les organisations.

7. Construction de ressources Web.

8. Développement de formations pour les utilisateurs.

9. Discussion/débat.

10. Étude de cas.

11. Examens.

12. Exemples expliqués en classe.

13. Exercices en classe. 
Employé pour: exercices pratiques en classe; petits exercices pratiques en classe; travaux de groupe (en classe).

14. Exposés magistraux.

Employé pour: cours magistraux; enseignement magistral.

15. Introduction au travail en équipe.

16. Lectures.

Employé pour: lectures exploratoires; lectures obligatoires.

17. Observation d'une activité de formation avec une grille d'observation.

18. Plan d'information intégré (travail de synthèse).

19. Présentation des étudiants devant public.

20. Questions populaires.

21. Jeu questionnaire.

22. Rapport d'étonnement.

23. Rapport d'évaluation (travail de session).

24. Recherche commanditée d'information.

Employé pour: recherche commanditée; semaine des bases de données.

25. Séminaire.

Employé pour: séminaire oral.

26. Table ronde.

27. Travail de recherche.

Employé pour: dossier; travail de réflexion; travail de session écrit.

28. Travaux pratiques.

Employé pour: exercices en laboratoire; travail d'élaboration d'un plan de classification pour les archives définitives; travail en laboratoire.

29. Travaux sur l'actualité.

Employé pour: archivactualité; constitution de dossiers d'actualité; information numérique et actualité.

30. Visite de milieu.

Employé pour: rencontre d'archivistes dans leur milieu; visite en milieu de travail; visite en milieu professionnel.

\section{Annexe 3. Exemples de deux fiches annotées sur les pratiques pédagogiques utilisées à l'EBSI}

\section{Questions populaires}

Employé pour: Nil

\section{Description:}

Les étudiants sont appelés à élaborer des questions à développement sur toute la matière (deux questions par étudiant), qui pourront faire partie des questions d'examen. Les questions qui reviennent souvent sont retenues par l'enseignant. Les étudiants peuvent discuter de leurs questions en groupe avant de les soumettre à l'enseignant.

\section{Contexte d'utilisation:}

ARV1055 Organisation intellectuelle 2 - Certificat en archivistique (Isabelle Dion).

\section{Application:}

Taille de l'équipe: Ne s'applique pas.

Temps de réalisation: Ne s'applique pas.

Lieu de réalisation: À la maison.

Superviseurs/assistants: Ne s'applique pas.

Corrigé disponible: Ne s'applique pas.

Fréquence dans le cours: 2 fois.

Évaluation de l'apprentissage:

Cette pratique pédagogique n'est pas une méthode d'évaluation en soi. Cependant, les deux questions retenues figureront à l'examen final.

\section{Évaluation de la pratique:}

Pratique non évaluée, mais appréciée par les étudiants.

\section{Avantages:}

Cela permet aux étudiants de connaître à l'avance deux questions de l'examen, qu'ils préparent. Pour l'enseignant, cette pratique permet de constituer une base de questions potentielles pour les examens. Elle permet aussi de vérifier l'intérêt des étudiants pour la matière: les questions proposées par les étudiants portent souvent sur ce qu'ils ont bien compris.

\section{Limites:}

Requiert un groupe qui veut participer.

\section{Recommandations:}

Bien expliquer au départ les «balises» pour bien formuler la question posée, ce qui permet d'obtenir un ensemble homogène de questions.

Lien avec d'autres pratiques pédagogiques:

Lien avec l'examen.

\section{Visite de milieu}
Employé pour:
$\triangleright$ Rencontre d'archivistes dans leur milieu.
$\triangleright$ Visite en milieu de travail.
$\triangleright$ Visite en milieu professionnel. 


\section{Description:}

La visite de milieu s'inscrit dans l'utilisation des ressources du milieu. «Par ressources du milieu, nous entendons tant les personnes qui peuvent être invitées ou visitées que les sorties à l'extérieur, les richesses de l'environnement, les artefacts... " (Chamberland et al.:140.) Il s'agit donc de l'«utilisation et [ de l']exploitation des sources possibles d'apprentissage qu'offre le milieu» (Ibid.:139).

Cette pratique pédagogique consiste, pour les étudiants, à se rendre dans un milieu de travail pour y rencontrer les professionnels et avoir un aperçu concret de la réalité de leur travail. Les visites sont choisies en fonction des institutions et des aspects reliés à la matière du cours (par ex.: BLT6308, BLT6336). L'objectif est d'établir les liens essentiels entre la théorie et la pratique (par ex.: BLT6059).

Quelques exemples de milieux visités:

Visite d'une salle de montre (salle de nouveautés) (par ex.: chez Renaud-Bray, réservé aux entreprises) (BLT6122).

Visite d'un service d'archives et rencontre avec un ou des archivistes (BLT6059).

Visite de l'Office national du film, de Radio-Canada (BLT6308, BLT6336).

Dans le cas des cours BLT6308 et BLT6336, l'enseignant fait d'abord la visite seul pour avoir un aperçu de la situation. Pendant la visite avec les étudiants, il pose aussi des questions aux professionnels de l'information qui les accueillent, afin de s'assurer que les étudiants aient des renseignements sur des aspects précis.

Pour le cours BLT 6059, les étudiants passent une demi-journée avec un archiviste et son équipe, posent des questions, relèvent les forces et les lacunes du milieu, tout cela dans l'objectif de comprendre le profil de l'archiviste. Cette rencontre, donc, va plus loin qu'une visite.

\section{Contexte d'utilisation:}

BLT6059 Archivistique et information documentaire - M1.

BLT6122 Développement et gestion des collections - M2.

BLT6308 Documents visuels et sonores - M2.

BLT6336 Multimédia et hypermédia - M2.

\section{Application:}

Taille de l'équipe:

$\triangleright$ La classe est divisée en groupes de 12 étudiants pour la visite (BLT6059).

$\triangleright$ La classe est divisée en deux groupes pour la visite (BLT6122).

$\triangleright$ La classe peut être divisée en deux groupes, selon le nombre d'étudiants inscrits et des capacités d'accueil de l'institution qui reçoit (BLT6308, BLT6336).

Temps de réalisation:

$\triangleright 2$ heures à 2 heures et demie, au cours de la deuxième moitié de la session (BLT6059).

$\triangleright$ 30 minutes (BLT6122).

$\triangleright 1$ heure à 1 heure et demie (BLT6308, BLT6336).

Lieu de réalisation:

$\triangleright$ Service d'archives (BLT6059).

$\triangleright$ Salle de montre (BLT6122).

$\triangleright$ Office national du film, Radio-Canada (BLT6308, BLT6336).

Superviseurs/assistants: Ne s'applique pas.

$\triangleright$ Corrigé disponible: Ne s'applique pas.

$\triangleright$ Fréquence dans le cours: 1 fois (BLT6059, BLT6122, BLT6308, BLT6336).

\section{Évaluation de l'apprentissage:}

De manière générale, il s'agit d'une activité obligatoire pour les étudiants qui sont notés pour leur participation, ou encore pour leur participation et la rédaction d'un rapport de visite.

Voici quelques exemples d'évaluation:

$\triangle$ BLT6059: Activité obligatoire (5\%). Une liste d'éléments à recueillir durant la visite est communiquée aux étudiants avant la visite. Cela sert à rédiger un bref rapport.

$\triangleright$ BLT6122: $5 \%$ de la note finale (5 points pour la présence).

$\triangleright$ BLT6308, BLT6336: Ce n'est pas une méthode d'évaluation, mais les étudiants sont pénalisés s'ils n'y vont pas (l'enseignant consacre des points à la participation, dont il peut se servir de cette manière).

Correction faite par: l'enseignant.

Correction faite selon:

$\triangleright$ Participation à la visite (BLT6122, BLT6308, BLT6336).

$\triangle$ Critères prédéfinis servant à la rédaction d'un rapport de visite (BLT6059).

\section{Évaluation de la pratique:}

Cette pratique pédagogique est évaluée par les étudiants au moment de l'évaluation générale du cours à la fin de la session (BLT6122).

Certains enseignants font aussi une évaluation de cette pratique avec leurs étudiants:

$\triangleright$ L'enseignant demande aux étudiants s'ils aimeraient retourner dans ce milieu l'année suivante. Cette activité est appréciée par les étudiants (BLT6059).

$\triangleright$ Évaluation informelle avec les étudiants en classe (BLT6308, BLT6336). 


\section{Avantages:}

Les visites permettent aux étudiants de créer des liens avec les professionnels. Elles leur permettent aussi, dans certains cas, de faire du réseautage qui peut leur être bénéfique par la suite. Ainsi, cela a permis à certains étudiants de se procurer un emploi après avoir postulé à ces endroits, ou encore parce que les professionnels de ces milieux ont demandé à l'enseignant de leur recommander des noms d'étudiants pour des emplois.

De plus, cela assure le maintien d'une collaboration entre l'EBSI et les institutions.

Les visites donnent aux étudiants un aperçu de ce qui les attend sur le marché du travail, tout en leur donnant accès à un espace normalement réservé aux professionnels (donc non public) (par ex.: BLT6122). Cela permet aux étudiants de voir évoluer les professionnels dans leur milieu de travail, de constater les forces et faiblesses du milieu, de comprendre le profil de l'archiviste et de mieux connaître la profession (par ex.: BLT6059).

Les visites permettent de faire le pont entre la théorie et la pratique, puisque les étudiants voient concrètement comment les pratiques sont appliquées.

\section{Limites:}

Le succès des visites repose sur le professionnel du milieu chargé de faire la présentation au groupe d'étudiants. Il est important que l'enseignant soit présent durant la visite afin d'assurer un suivi de cette activité en classe.

Certains étudiants peuvent manifester peu d'intérêt pour les visites, en particulier si le milieu n'est pas «spectaculaire». Ainsi, une salle de montre peut leur paraître trop semblable à des étagères de librairie (par ex.: BLT6122).

La contrainte du nombre d'étudiants par groupe dépend du nombre d'étudiants total et des capacités d'accueil de l'institution qui reçoit. Il peut y avoir un problème de nombre d'étudiants par groupe: à plus de 12 étudiants, il devient difficile de parler aux gens du milieu et l'activité risque alors se transformer en simple visite.

Il faut augmenter le nombre de milieux à visiter (par ex.: BLT6059).

Il faudrait pouvoir rester dans les institutions plus longtemps pour pouvoir tout voir et pour pouvoir manipuler tous les outils tels que les bases de données (par ex.: BLT6308, BLT6336).

Il y a aussi les contraintes du temps de transport à l'aller et au retour, car les milieux sont parfois assez loin de l'EBSI, et il faut tenir compte des autres cours que suivent les étudiants avant et/ou après la visite.

\section{Recommandations:}

L'enseignant doit donner aux étudiants une bonne présentation du milieu avant de s'y rendre, et il est important qu'il participe lui aussi à la visite.

Il est important de faire un choix judicieux du milieu et du professionnel qui fait faire la visite.

Il faut se tourner vers des diplômés de l'EBSI. Pour cela, il est nécessaire de garder de bons contacts avec les milieux de travail en tout temps. L'EBSI a ceci de caractéristique qu'elle maintient beaucoup de liens avec ses diplômés, qu'il y a plusieurs occasions de fidélisation des diplômés, qu'il règne un esprit collégial et familial (notamment en raison de la langue) qui n'existe pas nécessairement aux États-Unis par exemple.

\section{Lien avec d'autres pratiques pédagogiques:}

Liens avec les pratiques suivantes:

$\triangleright$ Un autre type de rencontres avec des professionnels (invités en classe), pour atteindre le même objectif: comprendre le profil de l'archiviste (BLT 6059).

$\triangleright$ Exposés magistraux (BLT 6308, BLT 6336).

$\triangleright$ Exercices en classe (BLT 6308, BLT 6336).

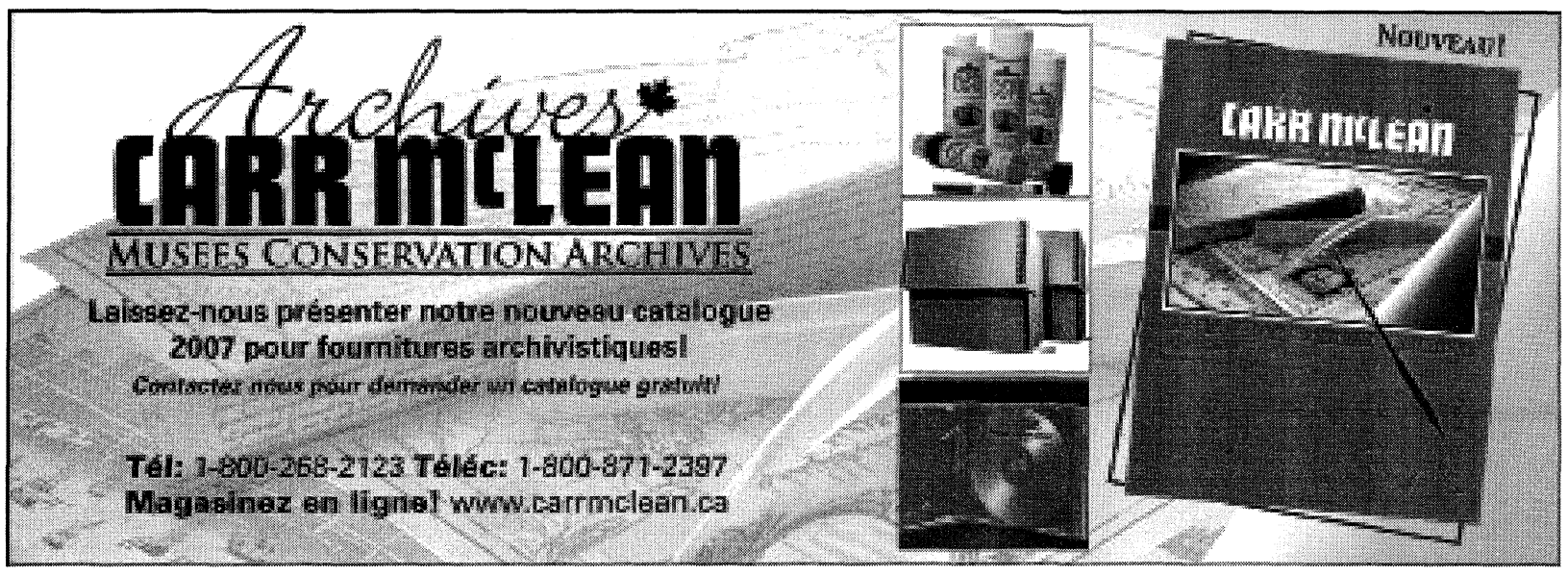

26 | JANVIER • MARS 2007 | DOCUMENTATION ET BIBLIOTHÈQUES 